\title{
The Relationship between Lifestyle and Costs Related to Medicine Use in Adults
}

\author{
Romulo Araujo Fernandes, ${ }^{1,2}$ Alessandra Madia Mantovani, ${ }^{1 \odot}$ Jamile Sanches Codogno, ${ }^{1,2}$ Bruna Camilo Turi-Lynch, ${ }^{1 \odot}$ \\ Subhash Pokhrel, ${ }^{3(10}$ Nana Anokye ${ }^{3(0)}$ \\ Programa de Pós-Graduação em Ciências da Motricidade - Instituto de Biociências - Universidade Estadual Paulista (UNESP), ${ }^{1}$ Rio Claro, SP - Brazil \\ Departmento de Educação Física - Faculdade de Ciências e Tecnologia - Universidade Estadual Paulista (UNESP), ${ }^{2}$ Presidente Prudente, SP - Brazil \\ Institute of Environment - Health and Societies - Brunel University - Uxbridge, ${ }^{3}$ London - United Kingdom
}

\begin{abstract}
Background: The unhealthy lifestyle is growing and this can have repercussions on health status demanding actions on the occurrence of diseases and leads to increased expenses.

Objective: To examine the interrelationship between the costs of medicine use and lifestyle behaviors.

Methods: A cohort study with 118 participants, age around $51.7 \pm 7.1$ years old. It was collected personal and anthropometric data and information about medicine of continuous use to calculate the costs. Lifestyle variables included habitual physical activity (PA) assessed by pedometer, sedentary behavior by Baecke questionnaire, sleep quality by mini sleep questionnaire and self-report of smoke and alcohol consumption. Statistical analyses were performed by BioEstat (version 5.2) and the significance level set at $p$-value $<0.05$.

Results: In 12 months, 62 subjects bought 172 medicines, representing an overall cost of US\$ 3,087.01. Expenditures with drugs were negatively related to $\mathrm{PA}(r=-0.194$, $p$-value $=0.035$ and $r=-0.281, p$-value $=0.002)$, but positively related with sleep quality $(r=0.299, p$-value $=0.001$ and $r=0.315$, $p$-value $=0.001)$ and age $(r=0.274, p$-value $=0.003)$. Four multivariate models were executed considering lifestyle behaviors in different moments of cohort and medicine costs, and all these models identify important relationship between lifestyle behaviors with expenditures with drugs.
\end{abstract}

Conclusion: Worse sleep quality seems to increase the costs related to medicine use in adults, while obesity and ageing play a relevant role in this phenomenon and alcohol consumption seems a variable with relevant economic impact. (Arq Bras Cardiol. 2019; 112(6):749-755)

Keywords: Quality of Life; Sedentary Lifestyle; Obesity; Sports Medicine; Longevity; Health Behavior, Exercíse.

\section{Introduction}

Over the last decades, the occurrence of obesity and chronic diseases has increased dramatically among adults worldwide. ${ }^{1}$ On the other hand, advances in medical sciences, development of new generation of medicines/therapies, have significantly improved quality of life and longevity. ${ }^{2,3}$

In developing countries, the use of any medicine is reported by $60 \%$ of the adult population, while the use of three or more medicines in the last two weeks is reported by about $18 \%$ of the population. ${ }^{4} \mathrm{~A}$ similar pattern is observed in Central Eastern Europe where more than 20\% of adults (18 years plus) report three or more medicine use. ${ }^{5}$ Narayan et al. ${ }^{3}$ found that in a period of nine years (from 2005 to 2013) the use of drugs for prevention purposes (aspirin, clopidogrel, statins and bisphosphonates) increased

Mailing Address: Rômulo Araújo Fernandes •

Roberto Simonsen Avenue, 305. Centro Educacional. Postal Code 19060-900. Presidente Prudente, SP - Brazil

Email: romulo_ef@yahoo.com.br

Manuscript received May 25, 2018, revised manuscript September 18, 2018, accepted September 19, 2018

DOI: $10.5935 / a b c .20190049$ significantly among New Zealand adults aged 65 years or more (about $19.5 \%, 2.9 \%, 7 \%$ and $2.3 \%$, respectively).

The dramatic rise in the prevalence of obesity and its associations with the development of metabolic and cardiovascular diseases would explain, at least in part, this increase trend. ${ }^{1,6}$ In fact, wide access to medicines by population signifies an improvement in the prevention/treatment of diseases. ${ }^{2}$ However, the potential adverse drug reactions generated by the use of unprescribed medicines constitute a global public health concern related to high healthcare costs. ${ }^{7,8}$

The economic burden related to medicine use involves not only health care costs resulting from inappropriate medication but also the purchase of prescribed and unprescribed medicines (public and out of pocket expenses). ${ }^{7,9}$ For example, in a period of five years from 2000 to 2004, the Brazilian Ministry of Health expended US\$ 916 million in programs to provide high-cost drugs to population. ${ }^{10}$ Despite the increasing economic burden related to medicines use, little is known about its underlying determinants. We need to know these determinants in order to identify target areas for policy making on managing health budgets, particularly in health systems in developing settings.

Unhealthy lifestyle behaviors (alcohol consumption, smoking, poor sleep habits and sedentary behaviors [SB]) have 
been shown to play an important role in the development of many diseases, ${ }^{11}$ but their direct relationship with the costs related to medicines use is unclear. For example, the occurrence of sleep disorders is highly prevalent in adults, ${ }^{12}$ but its economic burden is unknown. ${ }^{13} \mathrm{~A}$ longitudinal study carried out with 11,698 American employees identified that how worse were the sleep disorder reported the health care costs to increase in average US\$ $725.15 .{ }^{13}$ Similarly, in a 12-months study carried out in Taiwan, adults with positive diagnosis to obstructive sleep apnea were $66 \%$ more expensive (in terms of physician diagnoses, medications, treatments, surgeries, laboratory tests and diagnostic imaging) than those adults without the same diagnosis (US\$ $1,734.10$ versus US $\$ 1,041.30$, respectively). ${ }^{14}$ Conversely, improved levels of physical activity (PA) could reduce costs related to medicine use in adults, ${ }^{15,16}$ but its role in the potential relationship between unhealthy lifestyle behaviors and costs of medicine use has not been studied to date.

In this study, we examine the interrelationship between costs of medicine use and lifestyle behaviors (both healthy and unhealthy).

\section{Methods}

\section{Sample}

The data comes from a cohort study carried out in the city of Presidente Prudente which presents human development index $0.806,{ }^{17}$ placed on western Sao Paulo State (which is the state of the most industrialized Brazilian federation) from February/June 2014 (baseline) to May/December 2015 (follow-up). Sample size estimation was based on an equation for the correlation coefficient. Due to the absence of specific data about the relationship between lifestyle behaviors and health care costs in Brazil, ${ }^{16,18}$ we have adopted a correlation coefficient of 0.30 between PA and health care costs, ${ }^{16,18} z=1.96$ and power of $80 \%$ (adopting the abovementioned parameters, the minimum sample size required for this study was 86 participants). The inclusion criteria for participants were: 40-65 years old, no diagnosis of previous cardiovascular complications (e.g. stroke, heart attack), no diabetes complications (amputation or visual problems), no regular medication use, and no physical disability.

Invitation to participate in the study was conducted using advertisements (ie posters) in the Sao Paulo State University in Presidente Prudente and gyms/fitness centers across the city. Interested participants contacted the research staff, who then checked the profile of the participants against the inclusion criteria (participants who met all the inclusion criteria signed a written consent form). One hundred ninety-eight adults contacted the research staff and were considered eligible and undertook baseline assessment. The analysis herein covered 118 subjects (44 men and 74 women) assessed at both baseline and follow-up (12 months later). The excluded people were due to (a) dropouts $(n=62)$ and $(b)$ provision of less than seven days of pedometer use at baseline $(n=18)$.

All procedures (questionnaires, pedometers and body composition assessment) were performed by trained staff of researchers (Professors, MSc and PhD students) following the protocols of the Laboratory of Investigation in Exercise (LIVE), Brazil. ${ }^{19}$ The Ethics committee of the Sao Paulo State University (UNESP), campus of Presidente Prudente, approved the study.

\section{Costs of medicines use}

At baseline, the participants were given a questionnaire (in diary form) for medicine use and instructions (further clarification offered face to face by research staff) on how to fulfil the questionnaire. The participants reported the following data: (a) number and type of all medicines (prescribed and unprescribed); (b) how they obtained the medicines - through the Brazilian National Health System [BNHS] or out of pocket expenditure. The diary was filled for each of the 12 months of the cohort study. At the end of the follow-up period, the research staff collected back the completed diaries (Table 1). To calculate the cost of medicines, we used national prices presented by BNHS (for medicines delivered by the BNHS) and market prices from drug stores in the study area (medicines obtained via personal expenses). Costs were computed in Brazilian currency (Real\$) and converted to US dollar (US\$) using the cambial information provided by the Central Bank of Brazil.

\section{Lifestyle behavioral variables}

PA was measured using both objective and subjective measures at baseline and follow-up. Objective measure of PA was collected using pedometers (Yamax digiwalker, SW200 model, Japan), and specified in terms of step count. At both assessments periods (baseline and follow-up), pedometers were worn by participants for seven consecutive days. The pedometers were fixed laterally at the hip and were taken off only during periods of sleep and water-based activities. Participants logged (at the end of each day) the total step count. In the present study, PA denoted the number of days (out of 14 days assessed) that $\geq 7,500$ steps were achieved. In line with Tudor-Locke et al., ${ }^{20}$ participants who reached $\geq 7,500$ steps/day were classified as "sufficiently active". The subjective measure of PA was collected using Baecke's questionnaire. ${ }^{21}$ The questionnaire is composed of 16 questions about three PA domains (occupational, sports participation and leisure-time PA).

Data on SB at work (both baseline and follow up) were captured using the following question: "At work I sit"...; potential responses were: never [score attributed $=1$ ], seldom [score attributed $=2$ ], sometimes [score attributed $=3$ ], often [score attributed $=4$ ] and very often [score attributed $=5]$ ).

Quality of sleep was assessed at baseline and follow up using the Mini-Sleep Questionnaire, ${ }^{22}$ which includes 10 questions, each one with seven possible answers (ranging from never to always). The sum of these 10 answers generates a numerical score ranging from 10 to 70 points (higher scores indicate worse sleep quality).

Participants also self-reported at baseline and follow-up smoking status (yes or no current smoker) and weekly alcohol consumption (number of days per week with alcohol consumption). 


\begin{tabular}{lcc}
\hline Anatomical Therapeutic Chemical Code & Types of medicine & Number of medicines bought \\
\hline Digestive tract and metabolism & 18 & 33 \\
Blood and Blood-forming organs & 2 & 2 \\
Cardiovascular system & 31 & 42 \\
Dermatological & 1 & 1 \\
Genito-urinary system and sex hormones & 9 & 25 \\
Hormones, except sexual and insulin & 10 & 10 \\
Systemic anti-infective agents & 1 & 1 \\
Anti-neoplastics and immune modulators & 1 & 2 \\
Muscle-skeletal system & 7 & 9 \\
Nervous system & 28 & 38 \\
Antiparasitics & 1 & 2 \\
Respiratory system & 3 & 3 \\
Sensory organs & 1 & 1 \\
Other & 1 & 3 \\
Overall & 114 & 172 \\
\hline
\end{tabular}

\section{Covariates}

Covariates were data collected via questionnaire (sex [male or female], date of birth [chronological age estimated using the difference between birthday and date of assessment] and formal schooling [in years]). Clinical data also were evaluated (body fatness [dual-energy X-ray absorptiometry], systolic and diastolic blood pressure respectively). Researchers performed the clinical measures in university facilities with controlled temperature and followed standardized procedures.

\section{Statistical analyses}

Descriptive statistics were undertaken using mean, 95\% confidence intervals $(95 \% \mathrm{Cl})$ and proportions as appropriate. Due to non-parametric distribution (attested by KolmogorovSmirnov test), the costs of medicine use were converted into base-10 logarithms.

Both the Pearson correlation and linear regression were conducted to assess the relationship between the costs of medicines and the independent variables. In the former approach, Pearson correlation (expressed as standardized coefficients [" $r$ " values]) analyzed the relationship of the costs of medicine use with lifestyle behaviors (sleep quality, PA, SB at work, smoking and alcohol consumption) and covariates (sex, age, schooling, blood pressure and body fatness) separately. In the latter, linear regression models (expressed as unstandardized coefficients [ $\beta$ values]) were fitted to examine the relationship between the costs of medicine use and lifestyle behaviors controlling for all covariates. For each approach, four models were fitted based on different specifications of lifestyle behaviors ([A] only baseline values, $[B]$ only follow-up values, [C] difference between follow-up and baseline and [D] sum of baseline and follow-up), to explore the differential relationship these specifications may present. Diagnosis of multicollinearity and homoscedasticity were assessed and the linear regression models were considered adequately fit.
All analyzes were performed using BioEstat (version 5.2) and the significance level was set at $p$-value $<0.05$.

\section{Results}

At baseline, the mean age of the sample was $51.7 \pm 7.1$ years, ranging from 40 to 68 years (Table 2). Alcohol was consumed on average 2.1 days per week, while $5.1 \%$ of the sample were smokers. Expenses on medicine use were reported by $52.5 \%$ of the sample. During 12-months of follow-up, 62 subjects bought 172 medicines (Table 2), representing an overall cost of US\$3,087.01 for the entire sample. There was no missing data.

PA decreased significantly from baseline to follow-up $(p$-value $=0.024)$, while the score for SB at work $(p$-value $=0.396)$, sleep quality $(p$-value $=0.951)$ and alcohol consumption $(p$-value $=0.100)$ remained stable between baseline and follow-up.

In the bivariate analysis, costs of medicine use were negatively related to $\mathrm{PA}_{\text {baseline }}(r=-0.194 ; \mathrm{p}$-value $=0.035)$, $\mathrm{PA}_{\text {follow-up }}(\mathrm{r}=-0.281 ; \mathrm{p}$-value $=0.002)$, but positively related with sleep quality baseline $(r=0.299 ; p$-value $=0.001)$ and sleep quality ${ }_{\text {follow-up }}(r=0.315 ; p$-value $=0.001)$, and age $_{\text {baseline }}(r=0.274 ; p$-value $=0.003)$. Gender, education, SB at work, alcohol consumption and smoking were not significantly related with costs of medicine use. There was no interrelationships among the lifestyle behaviors.

In the multivariate model considering lifestyle behaviors at baseline (Model-A), sleep quality and body fatness were positively related to higher 12-months medicine costs, while alcohol consumption was negatively related to it. Model-A explained $19.1 \%$ of all variance in the outcome (Table 3). In the multivariate model considering lifestyle behaviors at follow-up (Model-B), only sleep quality was positively related to higher 12-months medical costs. Model-B explained $21.9 \%$ of all variance in the medicine costs. 
Table 2 - Summarized characteristics of the sample $(n=118)$

\begin{tabular}{|c|c|c|}
\hline \multirow{2}{*}{ Variables } & \multicolumn{2}{|c|}{ Descriptive Statistic } \\
\hline & Mean $(95 \% \mathrm{Cl})$ & Median (IR) \\
\hline Age (years) $)_{\text {baseline }}$ & 51.7 (50.4 to 53.1$)$ & $51.1(10.1)$ \\
\hline Body weight $(\mathrm{kg})_{\text {baseline }}$ & 74.6 (71.7 to 77.4$)$ & $72.6(16.6)$ \\
\hline Height $(m)_{\text {baseline }}$ & 1.65 (1.63 to 1.67$)$ & $1.65(0.15)$ \\
\hline $\operatorname{BMI}\left(\mathrm{Kg} / \mathrm{m}^{2}\right)_{\text {baseline }}$ & 26.92 (26.3 to 27.5$)$ & $26.41(5.98)$ \\
\hline Costs with medicine (US\$) follow-up & 26.16 (17.7 to 34.62$)$ & $1.19(42.91)$ \\
\hline Alcohol consumption (days) ${ }_{\text {baseline }}$ & 2.1 (1.9 to 2.3$)$ & $2(2)$ \\
\hline Alcohol consumption (days) follow-up & $1.9(1.7$ to 2.2$)$ & $1(2)$ \\
\hline Sleep quality (MSQ score) $)_{\text {baseline }}$ & $26.6(24.7$ to 28.4$)$ & $25(13)$ \\
\hline Sleep quality (MSQ score) $)_{\text {follow-up }}$ & $26.8(24.8$ to 28.7$)$ & $26(15)$ \\
\hline $\mathrm{PA}(\geq 7,500 \text { steps/day) })_{\text {baseline }}$ & $2.3(1.8$ to 2.7$)$ & $1(5)$ \\
\hline $\mathrm{PA}(\geq 7,500 \text { steps/day })_{\text {follow-up }}$ & $1.9(1.5$ to 2.3$)$ & $1(4)$ \\
\hline SB at work (score) $)_{\text {baseline }}$ & 3.2 (2.9 to 3.4$)$ & $3(2)$ \\
\hline SB at work (score) $)_{\text {follow-up }}$ & 3.1 (2.9 to 3.3$)$ & $3(2)$ \\
\hline Smoking (yes [\%]) $)_{\text {baseline }}$ & $5.1 \%(1.1 \%$ to $9.1 \%)$ & -- \\
\hline Smoking (yes [\%]) & $5.9 \%(1.6 \%$ to $10.1 \%)$ & -- \\
\hline Medicine use (yes [\%]) follow-up & $52.5(43.5 \%$ to $61.5 \%)$ & --- \\
\hline
\end{tabular}

95\% CI: 95\% confidence interval; IR: interquartile range; BMI: body mass index; MSQ: mini-sleep questionnaire; PA: physical activity; SB: sedentary behavior.

Table 3 - Linear regression describing the relationship between 12-months medicine costs (dependent variable) and lifestyle behaviors ( $\mathrm{n}=118$ )

\begin{tabular}{|c|c|c|c|c|}
\hline & Model - A & Model - B & Model - C & Model - D \\
\hline \multirow[t]{2}{*}{ Independent variables } & Baseline & Follow-up & Follow-up minus Baseline & Baseline plus Follow-up \\
\hline & $\beta\left(\beta_{95 \% \mathrm{Cl}}\right)$ & $\beta\left(\beta_{95 \% \mathrm{Cl}}\right)$ & $\beta\left(\beta_{95 \% \mathrm{Cl}}\right)$ & $\beta\left(\beta_{95 \% \mathrm{Cl}}\right)$ \\
\hline PA (steps) & $-0.011(-0.078$ to 0.056$)$ & $-0.048(-0.122$ to 0.027$)$ & $-0.18(-0.092$ to 0.056$)$ & $-0.017(-0.058$ to 0.025$)$ \\
\hline Sleep quality (MSQ score) & $0.018(0.001$ to 0.034$)$ & $0.018(0.002$ to 0.033$)$ & $0.004(-0.017$ to 0.026$)$ & $0.011(0.002$ to 0.019$)$ \\
\hline Alcohol consumption (days) & $-0.137(-0.272$ to -0.002$)$ & $-0.111(-0.241$ to 0.019$)$ & $0.034(-0.140$ to 0.208$)$ & $-0.073(-0.145$ to -0.001$)$ \\
\hline Smoking (yes) & $0.157(-0.514$ to 0.828$)$ & $0.424(-0.224$ to 1.072$)$ & $0.819(-0.376$ to 2.014$)$ & $0.170(-0.172$ to 0.513$)$ \\
\hline SB at work (frequency) & $0.021(-0.114$ to 0.157$)$ & $0.017(-0.123$ to 0.157$)$ & $0.023(-0.154$ to 0.201$)$ & $0.006(-0.070$ to 0.082$)$ \\
\hline Sex (female) ${ }^{*}$ & $-0.447(-0.900$ to 0.007$)$ & $-0.341(-0.780$ to 0.098$)$ & $-0.302(-0.731$ to 0.126$)$ & $-0.421(-0.872$ to 0.029$)$ \\
\hline Age (years)* & $0.021(-0.004$ to 0.047$)$ & $0.020(-0.004$ to 0.044$)$ & $0.029(0.003$ to 0.056$)$ & $0.019(-0.005$ to 0.044$)$ \\
\hline Body fatness $(\%)^{*}$ & $0.023(0.003$ to 0.043$)$ & $0.019(-0.002$ to 0.039$)$ & $0.027(0.007$ to 0.048$)$ & $0.020(-0.001$ to 0.040$)$ \\
\hline $\mathrm{DBP}(\mathrm{mmHg})^{*}$ & $0.017(-0.010$ to 0.044$)$ & $0.021(-0.005$ to 0.047$)$ & $0.014(-0.014$ to 0.043$)$ & $0.020(-0.007$ to 0.046$)$ \\
\hline Schooling (years)* & $0.084(-0.024$ to 0.193$)$ & $0.091(-0.020$ to 0.202$)$ & $0.080(-0.026$ to 0.187$)$ & $0.092(-0.019$ to 0.202$)$ \\
\hline \multicolumn{5}{|l|}{ Linear regression parameters } \\
\hline $\mathrm{R}$ & 0.527 & 0.549 & 0.473 & 0.548 \\
\hline$r^{2}$ & 0.278 & 0.301 & 0.224 & 0.300 \\
\hline$r^{2}$ adjusted & 0.191 & 0.219 & 0.131 & 0.217 \\
\hline
\end{tabular}

*: only baseline values were used; Model-A: lifestyle behaviors inserted as baseline values; Model-B: lifestyle behaviors inserted as follow-up values; Model-C: lifestyle behaviors inserted as follow-up minus baseline values; Model-D: lifestyle behaviors inserted as baseline plus follow-up values; $95 \% \mathrm{Cl}$ : $95 \%$ confidence interval; PA: physical activity; MSQ: mini-sleep questionnaire; SB: sedentary behavior; SBP: systolic blood pressure; DBP: diastolic blood pressure. 
In the multivariate model considering changes over time in lifestyle behaviors (Model-C), age and body fatness were positively related to higher 12 -months medicine costs. Model-C explained $13.1 \%$ of all variance in the medicine costs. In Model-D (sum of baseline and follow-up values), sleep quality had a positive relationship with medicine use (Table 3). On the other hand, alcohol consumption was negatively related to costs of medicine use. Model-D explained 21.7\% of all variance in the medicine costs.

\section{Discussion}

This study shows that lifestyle behaviors particularly worse sleep quality leads to higher costs related to medicine use. Body fatness was also found to be an important predictor positive effect on costs. In overall, $52.5 \%$ of adults reported any medicine use during the cohort period, while $20.5 \%$ $(n=24)$ of these same adults reported the use of three or more medicines. These rates are similar to Brazilian (18\%) and European (20\%) surveys, in which population-based samplings were carried out. ${ }^{4,5}$

Another similarity with previous studies observed in our findings is that drugs for the treatment of cardiovascular diseases were the most reported by the participants. A study carried out in New Zealand examining the trends of medicine use in adults aged $\geq 65$ years from 2005 to 2013 identified that the use of drugs to prevent cardiovascular events (aspirin and statins) increased significantly. ${ }^{3}$ The dynamics observed for medicines to the treatment of cardiovascular diseases seems affected by aging as well (in our study, a relevant covariate in the multivariate models). Previous data have identified that consumption of aspirin and dipyridamole increased in older adults at a higher rate than observed in younger ones. ${ }^{3}$

The increased amount paid by older adults can be supported by the natural effects that ageing exert over organs of the human body and their functions, ${ }^{23}$ but also boosted by the reduced PA observed in older groups. ${ }^{24}$ In the analyzed sample, although the effect of age on costs with medicine use was not mediated by PA, they were related with other in crude analyzes, denoting the relevance of actions targeting the improvement of PA practice mainly in population groups composed of older adults. ${ }^{23,24}$

In this sample, the higher cost with medicines in adults with sleep disorders can represent not only the treatment of the sleep disorders itself, but also the use of medicines to relief its symptoms and hence to maintain the daily activities, such as work. ${ }^{13,25}$

The findings related to alcohol consumptions were surprising because usually the higher alcohol consumption is linked to higher health care costs, ${ }^{26,27}$ and not the opposite as observed in our study. In fact, the linkage between alcohol consumption and health care costs can be direct (e.g. diseases directly linked to alcohol consumption) and indirect (e.g. traffic car accident), but it is important to take into account that some kinds of alcoholic drinks have healthy characteristics, such as anti-inflammatory properties observed in the red wine. ${ }^{28}$ Therefore, the explanation for our interesting finding could be due to both the type and amount of alcohol consumed. However, our study looked at only the number of days per week with alcohol consumption, and not amount and type of alcohol consumed, which characterizes a limitation in our study.

Other limitations of the study are worth mentioning. The first limitation of the study is the small sample size. The current study has statistical power of $80 \%$ to detect coefficient of correlation of 0.256 or higher, while the relationship between some behaviors and costs with medicine are around $r=0.110 .{ }^{16}$ Even considering the fact that the inclusion of covariates increases the power of multivariate models, ${ }^{29}$ the reduced sample size may have been responsible for the absence of significant relationship between PA and costs with medicine. Another limitation related to objective measures of PA is the logging of data by the participants because every day they had to note the number of steps displayed in the pedometer. Although this method is widely used, ${ }^{19}$ it could have led to misreporting. As above mentioned, the absence of measures of amount and kind of alcoholic drinks and sedentary behavior (by screen time on TV or computer) constitute limitations as well. Further studies could explore the impact of these.

\section{Conclusions}

Worse sleep quality seems to increase the costs related to medicine use in adults, while obesity and ageing play a relevant role in this phenomenon. Moreover, alcohol consumption seems a variable with relevant economic impact, but further studies are necessary to identify clearly the direction of its relationship with medicine costs.

\section{Acknowledgment}

To the Fundação de Amparo a Pesquisa do Estado de São Paulo (FAPESP) (process number: 2017/50026-7 and 2015/20460-1) and to the Coordenação de Aperfeiçoamento de Pessoal de Nível Superior (CAPES).

\section{Author contributions}

Conception and design of the research: Fernandes RA; Acquisition of data and Analysis and interpretation of the data: Mantovani AM; Statistical analysis: Mantovani AM, Anokye N; Writing of the manuscript: Codogno JS, Turi-Lynch BC, Anokye $\mathrm{N}$; Critical revision of the manuscript for intellectual content: Codogno JS, Turi-Lynch BC, Pokhrel S.

\section{Potential Conflict of Interest}

No potential conflict of interest relevant to this article was reported.

\section{Sources of Funding}

There were no external funding sources for this study.

\section{Study Association}

This article is part of the thesis of Doctoral submitted by Alessandra Madia Mantovani, from Universidade Estadual Paulista. 


\section{Ethics approval and consent to participate}

This study was approved by the Ethics Committee of the Faculdade de Ciências e Tecnologia da Universidade Estadual Paulista under the protocol number 349.306/2013.

\section{References}

1. NCD Risk Factor Collaboration (NCD-RisC). Trends in adult body-mass index in 200 countries from 1975 to 2014: a pooled analysis of 1698 population-based measurement studies with $19 \cdot 2$ million participants. Lancet. 2016;387(10026):1377-96

2. Holloway KA, Henry D. WHO essential medicines policies and use in developing and transitional countries: an analysis of reported policy implementation and medicines use surveys. PLoS Med. 2014;11(9):e1001724.

3. Narayan SW, Tordoff JM, Nishtala PS. Temporal trends in the utilisation of preventive medicines by older people: A 9-year population-based study. Arch Gerontol Geriatr. 2016 Jan-Feb;62:103-11.

4. Bertoldi AD, Hallal PC, Barros AJ. Physical activity and medicine use: evidence from a population-based study. BMC Public Health. 2006 Sep $6 ; 6: 224$.

5. Vogler S, Österle A, Mayer S. Inequalities in medicine use in Central Eastern Europe: an empirical investigation of socioeconomic determinants in eight countries. Int J Equity Health. 2015 Nov 5;14:124.

6. Fernandes RA, Zanesco A. Early sport practice is related to lower prevalence of cardiovascular and metabolic outcomes in adults independently of overweight and current physical activity. Medicina (Kaunas). 2015;51(6):336-42 .

7. Hyttinen V, Jyrkkä J, Valtonen H. A systematic review of the impact of potentially inappropriate medication on health care utilization and costs among older adults. Med Care. 2016;54(10):950-64

8. White KG. UK interventions to control medicines wastage: a critical review. Int J Pharm Pract. 2010;18(3):131-40.

9. Arsenijevic J, Pavlova M, Rechel B, Groot W. Catastrophic health care expenditure among older people with chronic diseases in 15 european countries. PLoS One. 2016;11(7):e0157765.

10. Brandão CM, Guerra AA Jr, Cherchiglia ML, Andrade EL, Almeida AM, da Silva GD, et al. Expenses of the Brazilian Ministry of Health for high-cost drugs: a demographic and clinical analysis. Value Health. 2011;14(5 Suppl 1):S71-7.

11. Shi Z, Zhang T, Byles J, Martin S, Avery JC, Taylor AW. Food habits, lifestyle factors and mortality among oldest old chinese: The Chinese Longitudinal Healthy Longevity Survey (CLHLS). Nutrients. 2015;7(9):7562-79.

12. Zanuto EA, de Lima MC, de Araújo RG, da Silva EP, Anzolin CC, Araujo MY, et al. Sleep disturbances in adults in a city of Sao Paulo state. Rev Bras Epidemiol. 2015;18(1):42-53.

13. Hui SK, Grandner MA. Trouble sleeping associated with lower work performance and greater health care costs: Iongitudinal data from Kansas State Employee Wellness Program. J Occup Environ Med. 2015;57(10):1031-8.

14. Kao LT, Lee HC, Lin HC, Tsai MC, Chung SD. Healthcare service utilization by patients with obstructive sleep apnea: a population-based study. PLoS One. 2015;10(9):e0137459.

15. Codogno JS, Fernandes RA, Sarti FM, Freitas Júnior IF, Monteiro HL. The burden of physical activity on type 2 diabetes public healthcare expenditures among adults: a retrospective study. BMC Public Health. 2011 May 4;11:275.
All the procedures in this study were in accordance with the 1975 Helsinki Declaration, updated in 2013. Informed consent was obtained from all participants included in the study.
16. Codogno JS, Turi BC, Kemper HC, Fernandes RA, Christofaro DG, Monteiro $\mathrm{HL}$. Physical inactivity of adults and 1-year health care expenditures in Brazil. Int J Public Health. 2015;60(3):309-16.

17. Turi BC, Monteiro HL, Fernandes RA, CodognoJS. The impact of physical activity on mitigation of health care costs related to diabetes mellitus: findings from developed and developing settings. Curr Diabetes Rev. 2016;12(4):307-11.

18. Instituto Brasileiro De Geografia e Estatística (IBGE). Cidades e estados [mapa na internet]. Rio de Janeiro; [s.d.] [citado 11 set. 2018]. Disponível em: https://www.ibge.gov.br/estatisticas-novoportal/por-cidade-estadoestatisticas.html?t= destaques\&c $=3541406$.

19. Mantovani AM, Duncan S, Codogno JS, Lima MC, Fernandes RA.Differen amounts of physical activity measured by pedometer and the associations with health outcomes in adults. J Phys Act Health. 2016;13(11):1183-91.

20. Tudor-Locke C, Schuna JM Jr, Barreira TV, Mire EF, Broyles ST, Katzmarzyk PT, et al. Normative steps/day values for older adults: NHANES 2005-2006. J Gerontol A Biol Sci Med Sci. 2013;68(11):1426-32.

21. Baecke JA, Burema J, Frijters JE. A short questionnaire for the measurement of habitual physical activity in epidemiological studies. Am J Clin Nutr. 1982;36(5):936-42.

22. Falavigna A, de Souza Bezerra ML, Teles AR, Kleber FD, Velho MC, da Silva $\mathrm{RC}$, et al. Consistency and reliability of the Brazilian Portuguese version of the Mini-Sleep Questionnaire in undergraduate students. Sleep Breath. $2011 ; 15(3): 351-5$.

23. Wichi RB, De Angelis K, Jones L, Irigoyen MC. A brief review of chronic exercise intervention to prevent autonomic nervous system changes during the aging process. Clinics (Sao Paulo). 2009;64(3):253-8.

24. MilanovićZ, Pantelić S, Trajković N, Sporiš G, Kostić R, James N. Age-related decrease in physical activity and functional fitness among elderly men and women. Clin Interv Aging. 2013;8:549-56.

25. Araujo MY, Sarti FM, Fernandes RA, Monteiro HL, Turi BC, Anokye N, et al. Association between costs related to productivity loss and modified risk factors among users of the Brazilian National Health System. J Occup Environ Med. 2017;59(3):313-19.

26. Gómez-Restrepo C, Gómez-García MJ, Naranjo S, Rondón MA, AcostaHernández AL. Alcohol consumption as an incremental factor in health care costs for traffic accident victims: evidence in a medium sized Colombian city. Accid Anal Prev. 2014 Dec;73:269-73.

27. Neramitpitagkul P, Lertpitakpong C, Yothasamut J, Thavorncharoensap M Chaikledkaew U, Teerawattananon Y. Economic impact on health-care costs related to major diseases including HIV/AIDS due to alcohol drinking among Thai populations. Value Health. 2009;12(Suppl 3):S97-100.

28. Kwan HY, Chao X, Su T, Fu X, Tse AK, Fong WF, et al. The anticancer and antiobesity effects of Mediterranean diet. Crit Rev Food Sci Nutr. 2017;57(1):82-94.

29. Lingsma H, Roozenbeek B, Steyerberg E; IMPACT investigators. Covariate adjustment increases statistical power in randomized controlled trials. J Clin Epidemiol. 2010;63(12):1391. 
Lifestyle and costs of medicine use

Original Article 